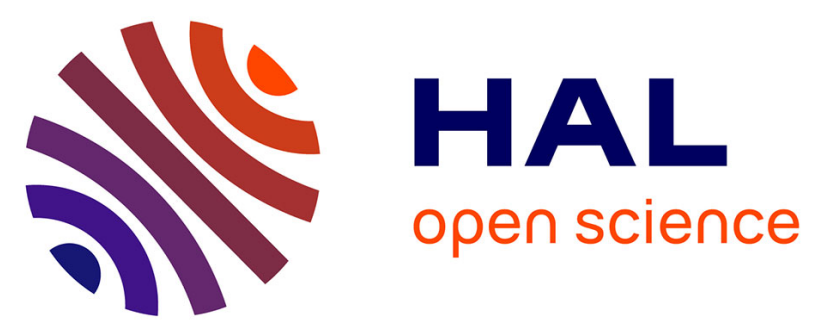

\title{
Fast contactless vibrating structure characterization using real time field programmable gate array-based digital signal processing: Demonstrations with a passive wireless acoustic delay line probe and vision
}

Gwenhaël Goavec-Merou, Nicolas Chretien, Jean-Michel Friedt, Patrick Sandoz, Gilles Martin, Michel Lenczner, Sylvain Ballandras

\section{To cite this version:}

Gwenhaël Goavec-Merou, Nicolas Chretien, Jean-Michel Friedt, Patrick Sandoz, Gilles Martin, et al.. Fast contactless vibrating structure characterization using real time field programmable gate arraybased digital signal processing: Demonstrations with a passive wireless acoustic delay line probe and vision. Review of Scientific Instruments, 2014, 85, pp.015109. 10.1063/1.4861190 . hal-01073841

\author{
HAL Id: hal-01073841 \\ https://hal.science/hal-01073841
}

Submitted on 10 Oct 2014

HAL is a multi-disciplinary open access archive for the deposit and dissemination of scientific research documents, whether they are published or not. The documents may come from teaching and research institutions in France or abroad, or from public or private research centers.
L'archive ouverte pluridisciplinaire HAL, est destinée au dépôt et à la diffusion de documents scientifiques de niveau recherche, publiés ou non, émanant des établissements d'enseignement et de recherche français ou étrangers, des laboratoires publics ou privés. 
AIP/123-QED

\section{Fast contactless vibrating structure characterization using real time FPGA-based digital signal processing: demonstrations with a passive wireless acoustic delay line probe and vision}

G. Goavec-Mérou, ${ }^{1}$ N. Chrétien, ${ }^{2}$ J.-M Friedt, ${ }^{2,}$ a) P. Sandoz, ${ }^{3}$ G. Martin, ${ }^{1}$ M. Lenczner, ${ }^{1}$ and S.Ballandras ${ }^{4}$

1) FEMTO-ST, Time \& frequency department, UMR CNRS 6174 ,

Univ. Franche Comté, Besançon, France

2) SENSeOR SAS, Besançon, France

3) FEMTO-ST, Applied Mechanics department, UMR CNRS 6174,

Univ. Franche-Comté, Besançon, France

4)Frec'n'sys SASU, Besançon, France

(Dated: 18 December 2013)

Vibrating mechanical structure characterization is demonstrated using contact-less techniques best suited for mobile and rotating equipments. Fast measurement rates are achieved using Field Programmable Gate Array (FPGA) devices as real-time digital signal processors. Two kinds of algorithms are implemented on FPGA and experimentally validated in the case of the vibrating tuning fork. A first application concerns in-plane displacement detection by vision with sampling rates above $10 \mathrm{kHz}$, thus reaching frequency ranges above the audio range. A second demonstration concerns pulsed-RADAR cooperative target phase detection and is applied to radiofrequency acoustic transducers used as passive wireless strain gauges. In this case, the $250 \mathrm{ksamples} / \mathrm{s}$ refresh rate achieved is only limited by the acoustic sensor design but not by the detection bandwidth. These realizations illustrate the efficiency, interest and potentialities of FPGA-based real-time digital signal processing for the contact-less interrogation of passive embedded probes with high refresh rates.

PACS numbers: 84.40.-x,43.20.Ye, 43.38.Rh

Keywords: strain gauge, wireless, battery-less, surface acoustic wave, RADAR, signal processing, image processing

\footnotetext{
a)Electronic mail: jmfriedt@femto-st.fr; http://jmfriedt.free.fr
} 


\section{INTRODUCTION}

Vibrating structure characterization is classically performed to assess mechanical equipment characteristics or as part of preventive maintenance. On rotating or moving parts, a wired solution is not robust for long term monitoring, with sliding contact or rotating optical couplers $^{1}$ being demonstrated in the literature for laboratory experiments. One alternative to structure bending and vibration analysis - whether applied to macroscopic ${ }^{2,3}$ or microscopic structures ${ }^{4,5}$ - is image processing ${ }^{6}$ in which the structure deformation and motion is detected either through motion of its natural surface properties (roughness yielding varying reflected light intensity) or artificially patterning the surface with a structure best suited to motion detection ${ }^{7}$. Embedding strain gauges on the mobile part with wireless communication yet on-board energy source ${ }^{8}$ reduces the life expectancy of the measurement setup to the battery life of the powered sensor and hence requires periodic maintenance ${ }^{9}$ : the broad field of wireless sensor networks (WSN) is concerned with such an approach ${ }^{10-13}$. Only very few studies are concerned with embedding passive sensors in the vibrating structure and using variations of the reflected signal when illuminated by RADAR signals ${ }^{14}$ - either through a RADAR Cross-Section (RCS) variation or resonance frequency variation ${ }^{15-17}$ - to measure strain in mechanical structures.

We here consider two contact-less vibrating structure characterization means: digital image processing and using piezoelectric acoustic delay lines acting as passive strain gauges probed through a wireless link. In the context of control, real time analysis of the recorded data is mandatory and postprocessing of large records is not an option. Furthermore, in the context of vibrational analysis, measurement speed is a significant indicator of sensor performance in order to extend to the highest vibration modes the analysis of the mechanical structure under investigation. As such, a real time fast measurement technique, as opposed to as stroboscopic approach yielding high equivalent sampling rate but assuming a periodic signal, allows for the detection of transient events with short durations ${ }^{18}$. While the envisioned algorithms are basic and have been described previously, the implementation on hardware compatible with real time feedback control is challenging, and is the topic of this paper.

Real time signal processing has long been devoted to analog processing, with the utmost bandwidth being achieved by piezoelectric and optical physical signal processing including 
convolution, correlation and Fourier transform ${ }^{19}$. The advent of digital signal processing yielded long term stability (no dependence of the analog component value to environmental conditions) and reconfigurability ${ }^{20}$ has become the mainstream implementation strategy, with the loss of real time signal processing when software and operating systems are involved. Field programmable gate arrays (FPGA) provide the means of achieving the best of both worlds, with digital signal processing running at radiofrequency rates. One current application of such an approach is concerned with Software Defined Radio aiming at sampling the baseband radiofrequency signal and performing all signal processing as software defined algorithms. In these demonstrations, we implement one example of digital image processing for motion detection with frame rates reaching the ksample rate (i.e. many thousand images recorded and processed every second) and a pulsed mode RADAR operating in the $2.45 \mathrm{GHz}$ aimed at probing a surface acoustic transducer acting as a passive, wireless strain sensor. The latter measurement technique is the result of a lengthy evolution ${ }^{21}$ in which a piezoelectric substrate is used to convert an incoming electromagnetic pulse to a mechanical (acoustic) wave: the environmental (temperature, strain) effect on the acoustic wave velocity modifies the time of flight of the acoustic pulse which, once converted back to an electromagnetic wave, is recorded by the RADAR receiver ${ }^{22}$. A measurement of the time of flight allows for the recovery of the acoustic velocity and hence the physical property most significantly affecting this velocity as selected by proper sensor design (minimizing unwanted physical property effect while emphasizing the dependence with the considered quantity). While most RADAR approaches have been developed with power consumption or computational limitations considerations, with the Frequency Modulated Continuous Wave approach $(\mathrm{FMCW})^{23}$ being the most basic implementation, high bandwidth measurements aimed at matching at best the performance of the sensor have not been presented in the literature. Our pulsed RADAR setup aims at using at best digital signal processing implemented on FPGA for performing a wireless measurement technique of probing acoustic delay lines, only limited in measurement bandwidth by the acoustic propagation time (physical measurement principle), and not by the means of measuring the signal. Pulse mode interrogation of wireless acoustic sensors has been demonstrated previously in academic settings ${ }^{24,25}$ but is still not used in commercial embodiments of such interrogation units applied to acoustic delay lines ${ }^{26}$. While passive target motion has been detected wirelessly with high bandwidth by using Doppler RADAR ${ }^{27,28}$, actual remote strain measurement has not been achieved 
by such means with the use of a dedicated transducer attached to the probed structure. Torque measurement using acoustic transducers acting as passive strain gauges attached to the structure under investigation has been investigated in-depth by Transense ${ }^{29,30}$ and $\mathrm{in}^{31}$.

\section{VISUAL DETECTION OF IN-PLANE DISPLACEMENTS}

\section{A. Measurement principle}

Optical measurement of vibrating structure is the most intuitive approach to contactless displacement detection. Various out of plane displacement measurement methods range from speckle interferometry detection to holographic displacement detection, while in-plane displacement is tackled by imaging ${ }^{32}$ and photogrametry. The latter approach will be of interest to us here, with the specific case of a quantitative displacement analysis by using a periodic pattern attached to the vibrating structure ${ }^{33,34}$ : the known periodicity yields a quantitative in-plane displacement measurement.

In-plane detection is usually performed as off-line processing due to the computationaly intensive processing requirements of correlation methods ${ }^{7}$. Furthermore, reaching sub-pixel resolution additionally requires some curve fitting or interpolation. We here first describe the basics of applying a cross-correlation method for detecting the motion of a patterned target (Fig. 1) fixed to the moving prong of a vibrating tuning fork ${ }^{33}$. We then demonstrate the implementation of this algorithm in a FPGA for real time computation of the target position.

For a given magnification factor, the analysis function is defined by the length $L$ of the sample (image length in pixels) and the period $p$ of the pattern (in pixels). We furthermore window the analysis function by a Gaussian envelope in order to generate the analysis function:

$$
\text { analysis }=\exp \left(-\left(\frac{x}{4 p \sqrt{2}}\right)^{2}\right) \times \exp \left(i \times x \frac{2 \pi}{p}+\varphi_{0}\right)
$$

with a pixel range $x=[1 . . L]-\bar{x}$ with $L$ the line length being analyzed and $\bar{x}$ the midpoint of this line. In our case, $L=1024, p=32$ and $\bar{x}=512 . \varphi_{0}$ is a constant selected to avoid $2 \pi$ rotation when computing the phase $\varphi$ of the cross-correlation which is performed in the spatial domain as $\varphi=\arg \left(\Sigma_{k=[1 . . L]}\right.$ measurement $_{k} \times$ analysis $\left._{k}\right)$. This phase is representative of the spatial position of the pattern, with a $2 \pi$ phase jumps associated with a one-period 
displacement of the reference grid. Hence, this analysis is quantitative with $p$ only given by the optical setup magnification factor and the actual grid periodicity. Typical resolutions

achieved by phase analysis and interpolating the unwrapped phase are in the range of $10^{-2}$ pixel or $10^{-3}$ grid period ${ }^{33}$, i.e. providing $10 \mathrm{~nm}$ positioning resolution when using visible illumination and low magnification lenses $(\times 10$ or $\times 20)$. This sub-pixel resolution is achieved by the integral (sum) over mulitple periods of the convolution of the image with the analysis function which has been tuned to match the period for a known pattern observed at a known magnification: the longer the observed line length (parameter $L$ ), the better the achievable resolution, for a given camera resolution and a given illumination condition (i.e. given signal to noise ratio of the recorded pixels).

\section{B. Real time processing: FPGA implementation and experimental results}

A Spartan 6 FPGA shares common data, address and control busses with an i.MX27 CPU as provided on a custom design based on the Armadeus Systems (Mulhouse, France) APF27 board. The FPGA is in charge of real time processing of the data as the pixels flow in through the serializer-deserializer interface, while the CPU is only used for post-processing the resulting data, namely computing the ratio of the imaginary to the real part of the complex output of the algorithm, computing the arctan of the result to reach an angle, and transfering the data to the user through an ethernet link.

[FIG. 1 about here.]

The imaging source is a PhotonFocus MVD1024E160 camera communicating through a CameraLink interface. Thus, the data source is the LVDS interface in charge of feeding the processing chain with 8-bit wide pixel values transferred as pairs at every clock cycle. Considering that the CameraLink digital interface clock speed is $80 \mathrm{MHz}$ and 2 bytes are provided at every clock cycle, the use of a 12-pixel wide (for averaging and thus noise reduction) and 1024-pixel long region of interest out of the available $1024 \times 1024$ pixel size yields a data transmission duration of $76.8 \mu$ s per frame. This camera hardware transfer time, in addition to the exposure duration, limits the frame rate. In addition, each line ends with a 100 ns delay, while before each frame the exposure time is set to the minimum value thanks to a bright LED light source, namely $10 \mu \mathrm{s}$. The practical frame rate meets the 
theoretical threshold of 5285 frame/s (Fig. 2), only limited by the camera data transfer rate since all processing operations are performed along the pipeline described in Fig. 1. This implementation is able to receive two consecutive pixels at each clock cycle and produce one phase output value one cycle after the end of image transmission. However, this analysis emphasizes a significant limitation due to the camera orientation since the pattern is varying along the columns. Each end of line induces a delay of $100 \mathrm{~ns}$ accumulating, for a 1024-line long image, up to $102.4 \mu \mathrm{s}$. Since each line tranfer completion requires a $100 \mathrm{~ns}$ delay, two implementations of the digital signal processing blocks are compared, one in which the pattern is along the acquisition line and one in which the pattern is along the acquisition column. Since the camera provides at each clock cycle adjacent pixels, the former approach illustrated in Fig. 1 (b) requires a common analysis coefficient while the latter apprach in Fig. 1 (c) requires two different analysis coefficients since adjacent pixels are not located on the same line. This difference yields some inversion in the multiplier and accumulator pattern as seen by comparing Figs. 1 (b) and (c).

By rotating the camera by an angle of $90^{\circ}$, the latencies due to the end of lines are reduced to the number of pixels parallel to each periodic pattern line. In our case, by averaging over 12 pixel wide lines, the $102.4 \mu$ s delay is reduced to $1.2 \mu \mathrm{s}$. Both approaches yield similar implementations and since phase computation derived from pixel averages and phase averaging computed on each column are linear operations, the two approaches yield results with the same resolution. However, with the latter approach, the frame rate increases up to 11360 fps. Removing the averaging along the 12-pixel wide line still increases the update rate up to $60610 \mathrm{fps}$, at the cost of reduced signal to noise ratio. In the latter case, the exposure duration of $10 \mu \mathrm{s}$ accounts for more than half of the frame acquisition duration and becomes the limiting factor defining the acquisition rate. The camera orientation allowing for maximum bandwidth is used to generate Fig. 3 .

The implementation of the signal processing block aims at using at best the parallel processing capability of a FPGA (Fig. 1, right): since two adjacent pixels are transferred by the camera at each clock cycle, the averaging is first performed by the adder. The multiplication by the analysis function is performed on the result, which is then accumulated to compute the cross-correlation function along a line. The computation is performed in parallel on the real part (I-component) and the imaginary part (Q-component), with only the analysis function changing from $\cos (2 \pi x / p)$ to $\sin (2 \pi x / p)$. 
Since the hardware multiplier and accumulator are optimized to operate on 18 bit values, the analysis function is scaled by 32765 to fit in a 16 bit signed integer value.

In order to avoid any limitation associated with digital data transfer to a general purpose $\mathrm{CPU}$, the sink of the data is a digital to analog (D/A) converter output providing voltages proportional to the I and Q components of the cross correlation. Fig. 2 exhibit oscilloscope outputs of these computations as a music tuning fork is vibrated at $440 \mathrm{~Hz}$ through a magnetic excitation by a voice coil ${ }^{32}$. The digital oscilloscope traces are here recorded and the phase extraction is performed as post-processing, avoiding the need to implement online the $\arctan ()$ lookup table function. In case of the use of such information for feedback control, the $2 \pi$ phase jump induced by the displacement of the target by more than one period must additionally be handled to avoid the discontinuity as observed on Figs. 2 and 4, right.

[FIG. 2 about here.]

Having achieved periodic oscillation condition observation of the tuning fork prong motion at a frequency known to be close to one of the eigenmodes of the device, we consider the impulse response of the tuning fork as observed by means of digital image processing (Fig. 3). In this case, two modes are well identified, the fundamental mode around $440 \mathrm{~Hz}$ and one overtone close to $10 \mathrm{kHz}$.

[FIG. 3 about here.]

We have previously described the implementation of a fast measurement algorithm identifying the limitation on the measurement bandwidth when using an acoustic resonator transducer acting as a strain gauge ${ }^{35}$ : a practical implementation reaching this refresh rate has been demonstrated by another group in ${ }^{36}$. Comparison of the resonator based measurement and the optical measurement, both of which are performed at $7 \mathrm{ksamples} / \mathrm{s}$, is provided in Fig. 4. The quantitative analysis of the resonator based strain gauge measurement to generate an estimate of the prong displacement has been provided previously ${ }^{35}$, while the optical periodic pattern image processing analysis directly provides a quantitative estimate of the prong end displacement. Fig. 4 exhibits on the one hand good agreement between the two measurement methods performed simultaneously, and emphasizes the limitation of 
the optical measurement when large displacements are considered, yielding $2 \pi$ phase rotations in the analysis (Fig. 4, right). For large displacements, the quartz strain gauge seems to overestimate the displacement, as has also been observed independently in quasi-static conditions, possibly due to the assumption on the tuning fork prong stress transfer to the piezoelectric substrate not being valid at high stress values. Indeed, in this test, the goldcoated backside of the acoustic resonator substrate was soldered using $\mathrm{SnPb}$ to the tuning fork, possibly inducing creep at high strain rates.

[FIG. 4 about here.]

\section{REAL TIME ACOUSTIC DELAY LINE PROBING}

\section{A. Pulsed-mode RADAR interrogation of an acoustic delay line}

Acoustic delay lines ${ }^{22}$ are one possible implementation of the broader range of acoustic sensors manufactured by patterning interdigitated electrodes on a single-crystal piezoelectric substrate. Various sensor designs - broadband delay lines, chirped delay lines, resonators exhibit various advantages and drawbacks, providing the freedom of selecting design rules depending on the targeted property. When considering vibrating structure monitoring, refresh rate appears as the most significant parameter, with interrogation range often a secondary issue. While a resonator configuration exhibits low loss and hence extended interrogation range in a wireless sensing application in which the resonance frequency is representative of the physical quantity under investigation and monitored through a radiofrequency link, the time constant for loading and unloading energy limits the update rate to a few kilohertz as was discussed earlier ${ }^{35}$ when operating in the $434 \mathrm{MHz}$ range, while the faster expected refresh rate achievable when using resonators in the $2450 \mathrm{MHz}$ range has not been demonstrated yet. On the other hand, an acoustic delay line converts an incoming electromagnetic pulse to an acoustic wave propagating on the single-crystal piezoelectric substrate at a velocity dependent on the physical property under investigation, and the two-way trip is recorded as a phase information of the reflection coefficient of the transducer probed through a wireless $\operatorname{link}^{37}$. The lack of energy confinement and the poor efficiency of electromechanical conversion define the acoustic delay line losses which are typically in the 35 to $40 \mathrm{~dB}$ range, hence reducing the interrogation range with respect to the resonator configuration (Fig 5). 
However, typical delay line tags exhibit a maximum echo delay of $4 \mu \mathrm{s}$, or a $1.6 \mathrm{~cm}$ two-way acoustic trip assuming a $4000 \mathrm{~m} / \mathrm{s}$ acoustic velocity on lithium niobate, and should thus be compatible with a $250 \mathrm{kHz}$ refresh rate. Indeed, in the most basic embodiment of a RADAR interrogation unit, a short (as will be defined later) radiofrequency pulse is generated, echoes are recorded up to the maximum duration of the acoustic delay, and this process is repeated. The repetition rate is thus only limited by the acoustic delay line length which defines the maximum delay. In this example, the 8-bit tag is assumed to reflect all incoming energy within the $4 \mu$ s after excitation by the incoming pulse, and we will demonstrate the practical implementation of a sampling strategy providing the maximum possible refresh rate.

[FIG. 5 about here.]

The emitted pulse length is defined in the time domain by the separation of the echoes, which itself is typically set in order to comply with radiofrequency emission regulation in the frequency domain. Since the $2.45 \mathrm{GHz}$ ISM band is $80 \mathrm{MHz}$ wide, typical pulse widths of $25 \mathrm{~ns}$ will occupy a $40 \mathrm{MHz}$ spectral width or half of the allocated band. In our experiments, $40 \mathrm{~ns}$ long pulses are generated as a tradeoff between radiofrequency switch toggling time constant (in our case, Minicircuits ZASWA-2-50DR+), maximizing transmitted energy, and separation of echoes. Ideally, the number of periods of the oscillating radiofrequency wave within the pulse should be equal to the number of interdigitated electrode pairs in the transducer. Although the FMCW approach has been classically selected for the low sampling rate of the receiver since audio-frequency beat signals between the emitted wave and the time-delayed echoes are generated, reaching the utmost bandwidth achievable by the transducer acoustic delay requires increasing the sweep rate, yielding increased beat frequency and hence increased sampling rate of the receiver analog to digital converter. Hence, whatever the considered architecture, high bandwidth signal processing necessarily requires high acquisition bandwidth, and we hence consider the pulsed mode RADAR approach as best suited to reach the maximum measurement speed since no additional processing (Fourier transform) is needed beyond recording the echoes received from the sensor.

\section{B. FPGA implementation and results}

The major implementation challenge of a fast reader is in recording the returned echoes, extracting the relevant information representative of the acoustic velocity, and repeating 
the process fast enough to provide the user with a fast refresh rate. Recent availability of fast analog to digital converters and arrays of reconfigurable logic gates as provided in FPGAs provides the basic tools for implementing the considered measurement technique (Fig. 6). The FPGA controls all interrogation sequences: an internal counter triggers the switch for generating a $40 \mathrm{~ns}$ pulse, the dataflow from the $1 \mathrm{GS} / \mathrm{s}$ analog to digital converter (ADC, Hittite HMCAD1511) is fed to a serializer-deserializer stage as available in the Xilinx Spartan6 FPGA, and either the full time domain record of 2048 samples is transferred to the iMX27 CPU for processing and display in order to select the echo delay, or the sample of interest is stored in RAM for further processing. This acquisition and processing strategy is reminiscent of the previous camera-based data acquisition approach. Notice that 2048 samples recorded at 500 Msamples/s provide a record lasting $5 \mu$ s or the total time delay of the acoustic transducer, while such a dataset fits within the RAMs internal to the FPGA. In the example we are demonstrating here, 2048 successive samples of the interrogation sequence are stored in RAM for display to the user as a delay line acting as a strain gauge glued on a $440 \mathrm{~Hz}$ musical tuning fork is probed. At $200 \mathrm{kHz}$ refresh rate, 2048 records of the I and Q components last $10.24 \mathrm{~ms}$ or about 4-periods of the vibrating tuning fork. This result is demonstrated in Fig. 7 in which a SAW delay line is glued on one prong of a tuning fork ${ }^{38}$ using Epotek H760E epoxy glue, hence illustrating the full speed measurement capability only limited by the delay line length. Faster refresh rates are accessible with shorter delay lines: since here an 8-bit tag is used the length is defined by the number of echoes, but sensing capability only does not require such long delay lines and shorter designs (with maximum echo delays of $500 \mathrm{~ns}$ for example) with only two reflectors as needed for a single physical quantity monitoring will provide higher refresh rates.

[FIG. 6 about here.]

Signal processing for physical quantity extraction is based on the classical phase detection of the echoes: since the two way trip of the acoustic wave along a path of length $2 d$ is $2 d / c$, the phase shift $\varphi$ with respect to the local oscillator (as obtained through the I/Q demodulator during the mixing process) is

$$
\varphi=2 \pi \cdot 2 d \cdot f / c
$$

where $f$ is the probed pulse central frequency. Getting rid of any drift of the local oscillator $(f)$ as well as of the distance between the reader and the sensor in case of mobile target 
applications requires a differential approach in which the phase difference between two echoes is processed. This computation is challenging since the phase extraction from the I and $Q$ components requires obtaining the arctan function, even though a feedback control aimed at keeping a constant strain with a quadrature condition only requires to keep the $Q$ component close to 0 and the $I$ component maximized, with no need to actually compute the associated phase. In the previous example, I and Q data were considered sufficient to provide an estimate of the acoustic velocity $c$ since over the short time scales of a few tens of milliseconds, the local oscillator drift is neglected.

\section{[FIG. 7 about here.]}

The need for high bandwidth measurement of vibrating structure mode identification is emphasized in Fig. 8 which exhibits the spectral analysis of the impulse response of the tuning fork fitted with the acoustic delay line strain gauge. In such a case, beyond the periodic analysis of the forced regime response of the tuning fork at its fundamental resonance frequency, high frequency modes are excited as well during the impulse response generation. As seen in the spectral analysis (Fig. 8, main graph) of the time-domain record (Fig. 8, insets), higher order modes ${ }^{38}$ are well observed up to $40700 \mathrm{kHz}$, at frequencies consistent with a tuning-fork emitted sound record sampled using a personal computer sound-card acquiring at a rate of $96 \mathrm{kS} / \mathrm{s}$. Identifying the prong motion characteristics of each one of these modes with respect to tabulated overtones is challenging due to the asymmetry between the two prongs introduced by the instrumentation process (adding the delay line transducer on the base of one prong and the target at the end of the other prong) which moves the resonance modes away from the expected frequencies modeled for a symetric tuning fork ${ }^{38}$. Nevertheless, a comparison of Figs. 8 and 3 illustrates consistency within the common frequency range (fundamental mode around $440 \mathrm{~Hz}$ and overtone around $10 \mathrm{kHz}$ ) and an inconsistency for a mode located around $5.5 \mathrm{kHz}$ which is observed by the SAW strain gauge (and the sound card records, not shown) but not by the optical methods. We attribute this mode to a non-flexural mode of the tuning fork, which is detected by acoustic means since the SAW strain gauge is sensitivite to multiple components of the strain field, while the image processing method is only sensitive to the displacement component orthogonal to the patterned lines. Hence, any displacement along the lines cannot be detected by the image processing method. 


\section{Strain measurement resolution assessment}

A significant hindrance of pulsed mode RADAR operating at temperature $T$ is the wide integration bandwidth $B$ which brings thermal noise $k_{B} \cdot T \cdot B$ on the low noise amplifier receiver stage, with $k_{B}=1.38 \cdot 10^{-23} \mathrm{~J} . \mathrm{K}^{-1}$ the Boltzman constant, over a much wider frequency range than FMCW or narrowband resonator based measurement techniques. One might wonder then whether the gain in refresh rate was achieved at the loss of resolution. In both FMCW and pulsed mode RADAR, the phase of the signal within the returned echoes is extracted by mixing with the local oscillator which has drifted over the propagation delay by a quantity $\sigma_{\varphi}$ defined as the phase noise of the local oscillator whose spectral density over a bandwidth $B W$ is defined as $S_{\varphi}=\sigma_{\varphi}^{2} / B W \operatorname{rad}^{2} / \mathrm{Hz}$.

Two conditions are considered: since the phase noise of a broadband amplifier (in our case the low noise receiver amplifier - LNA) rises with the received power, at close range the mixer output signal phase noise is defined by the local oscillator phase noise, while at longer range the received power is low enough that the LNA output phase noise drives the phase detection capability. In the latter case in which the receiver LNA overwhelms the local oscillator phase noise, a power $P$ received by an antenna connected to an amplifier at temperture $T$ and characterized by a noise factor $F$ induces a white phase noise floor of $S_{\varphi}=k_{B} \cdot T \cdot F / P$. Since no signal extraction is performed (e.g. using a cross correlation technique) to extract the returned signal from the noise, the received power is necessarily above the thermal noise power integrated over the analyzed bandwidth: in the case of a $500 \mathrm{MS} / \mathrm{s} \mathrm{A} / \mathrm{D}$ converter recording the $80 \mathrm{MHz}$-wide signal, $B W=40 \mathrm{MHz}$ and $k_{B} \times T \times B W=-98 \mathrm{dBm}$. In such a condition, the phase noise floor is $S_{\varphi}=-76 \mathrm{~dB} \cdot \mathrm{rad}^{2} / \mathrm{Hz}$ or a phase standard deviation

$\sigma_{\varphi}=\sqrt{S_{\varphi} \cdot B W}$, of $1.0 \mathrm{rad}$. Since the relative stress sensitivity along the propagation axis of lithium niobale $\mathrm{YXl} / 128^{\circ}$ cut we considered is ${ }^{39} d f / f=d c / c=-4.26 \mathrm{ppm} / \mathrm{MPa}$, operating at $2450 \mathrm{MHz}$ yields a phase to stress sensitivity $d \varphi=2 \pi \cdot 2 d \cdot f \cdot d c / c^{2}=2 \pi \cdot \tau \cdot d c / c$ where $\tau=2 d / c$ is the echo delay, so by normalizing with $\tau$ expressed in $\mu \mathrm{s}, d \varphi / \tau=$ $0.0656 \mathrm{rad} \cdot \mathrm{MPa}^{-1} \cdot \mu \mathrm{s}^{-1}$ and the measurement resolution at the detection limit of the returned signal is $1 / 0.0656=15 \mathrm{MPa}$ for an echo delayed by $1 \mu \mathrm{s}$. In the case of our experiment in which $+18.5 \mathrm{dBm}$ were emitted and $43 \mathrm{~dB}$ loss were exhibited by the acoustic delay line, the returned power to the first low noise amplifier is $-24.5 \mathrm{dBm}$, hence a phase resolution of $0.21 \mathrm{mrad}$ and the stress resolution is improved to $0.21 \cdot 10^{-3} / 0.0656=3.10^{-3} \mathrm{MPa}=3 \mathrm{kPa}$ 
or much less than the expected hundreds of $\mathrm{kPa}$ induced by the tuning fork vibration ${ }^{35}$.

In the case of a significant power returned by the sensor, the LNA phase noise is not the driving factor on the phase detection uncertainty which is then driven by the local oscillator. Assuming a flat, white, phase noise in the $100 \mathrm{kHz}$ (refresh rate and hence lower boundary of the integral limits) to above $10 \mathrm{MHz}$ (upper limit of the phase noise characterization) decade equal to a value $b_{0}$, then the phase fluctuation due to the local oscillator is $\sigma_{\varphi}=\sqrt{b_{0} \times B W}$. This analysis is numerically applied to the case of an Agilent E5071B network analyzer whose phase noise is measured (using an Agilent E5052B signal source analyzer) to be around $100 \mathrm{dBc} / \mathrm{Hz}$ in the $100 \mathrm{kHz}-1 \mathrm{MHz}$ region when providing a continuous output signal at $2.475 \mathrm{GHz}$ (0 Hz span). Under such considerations (signal to noise ratio being unity), the phase measurement resolution is $\sigma_{\varphi}=0.06 \mathrm{rad}$ and the stress resolution is $1 \mathrm{MPa}$, the poor performance being associated with a rather low quality source provided by the network analyzer. A better source exhibiting a noise floor of $b_{0}=-140 \mathrm{dBc} / \mathrm{Hz}$ would provide a resolution of $10 \mathrm{kPa}$.

It might be worth considering at which distance the transition from one regime to another occurs. The RADAR equation applied to a wireless acoustic sensor exhibiting accoustic loss $I L$ - acting as an equivalent RADAR cross section when combined with the signal wavelength to account for the antenna aperture - states that the receiver power $P_{R}$ is related to the emitted power $P_{E}$ when a target is located at distance $d$ from the reader by

$$
P_{R}=P_{E} \frac{\lambda^{4}}{I L \cdot(4 \pi)^{4}} \cdot \frac{1}{d^{4}}
$$

with $\lambda$ the incident pulse wavelength, so the transition distance occurs when the LNA phase noise $k_{B} T / P_{E}$ reaches the local oscillator noise $b_{0}: b_{0}=\frac{k_{B} \cdot T \cdot d^{4} \cdot I L \cdot\left(4 \pi^{4}\right)}{P_{E} \lambda^{4}}$ which is solved for a distance $d^{4}=\frac{P_{E} \cdot \lambda^{4} b_{0}}{k_{B} \cdot T \cdot I L \cdot(4 \pi)^{4}}$. A numerical application for $b_{0}=-100 \mathrm{dBc} / \mathrm{Hz}$ and $\lambda=12.2 \mathrm{~cm}$ shows that the critical distance is $d=12 \mathrm{~cm}$. A high quality oscillator exhibiting $b_{0}=$ $-150 \mathrm{dBc} / \mathrm{Hz}$ reduces this range further to $7 \mathrm{~cm}$, so the LNA will be the dominant issue in most practical applications. The maximum interrogation range is reached when the returned signal reaches the thermal noise, hence a distance $d$ so that $P_{R}=k_{B} \cdot T \cdot B W$ with again $B W=40 \mathrm{MHz}$ the transducer bandwidth, or $50 \mathrm{~cm}$ when emitting $P_{E}=10 \mathrm{~mW}$ (Fig. 9). This range extends to $88 \mathrm{~cm}$ when emitting $100 \mathrm{~mW}$ and $1.2 \mathrm{~m}$ if the insertion loss is reduced from $40 \mathrm{~dB}$ to $35 \mathrm{~dB}$. All these considerations assuming isotropic antennae fitting the interrogation unit and the sensor: practical applications must account for antenna gain 
which, at such a frequency, typically reaches $8.5 \mathrm{~dB}$ for antennae with $\lambda^{2}$ area (e.g HuberSuhner model SPA-2400, Switzerland) and additionnaly extends the interrogation range for dedicated setups (with the last numerical application, to $3.1 \mathrm{~m}$ ). Considering the $1 \%$ duty cycle, compliance with outdoor emission regulations in the $2.45 \mathrm{GHz}$ band (mean power of $10 \mathrm{dBm}$ ) allows for a peak power of $P_{E}=1 \mathrm{~W}$, yielding a $5.5 \mathrm{~m}$ expected measurement range, consistent with experimental demonstrations.

[FIG. 8 about here.]

[FIG. 9 about here.]

\section{CONCLUSION}

In the context of contactless vibrating structure characterization, we demonstrate real time image or radiofrequency signal processing for measuring displacements up to several $\mathrm{kHz}$ sampling rates with real time processing compatible with feedback control. The implementation of the pulsed mode RADAR with $500 \mathrm{MHz}$ sampling rate (2 ns sample period) allows for the identification of the velocity of a mechanical wave in an acoustic delay line acting as a passive, wireless sensor configuration with a measurement speed only limited by the acoustic wave echo delay. We demonstrate a $250 \mathrm{kHz}$ refresh rate, only limited by the $4 \mu$ s acoustic delay due to the $8 \mathrm{~mm}$ long acoustic path of the SAW tag used in this experiment, which could be increased further by using a dedicated SAW sensor design with smaller acoustic path length. Digital image processing for extracting pattern motion analysis using a cross-correlation technique was implemented digitally and achieved a refresh rate of $27 \mathrm{kHz}$. Both measurements performed on a same vibrating structure, a musical tuning fork, provided consistent vibrational mode analysis results. Real time performance yielding sub-10 $\mu$ s latencies when generating the computation results can only be achieved by implementing the processing algorithms in Field Programmable Gate Array (FPGA), with latencies only limited by the physical measurement principle (image acquisition or acoustic delay line probing) and not the computation time. 


\section{ACKNOWLEDGEMENTS}

Funding for this research was partly provided by the European Territorial Cooperation Programme INTERREG IV A France-Switzerland 2007-2013 and the OscillatorIMP Labex grant. The MIMENTO technological facility of FEMTO-ST, part of the RENATECH network, is acknowledged for providing technical support, especially V. Petrini for skillful handling of the microsystem transducers. The SAW delay lines were manufactured by É. Courjon at Frec'n'Sys SASU (Besançon, France). E. Foltête (Applied Mechanics department, FEMTO-ST, Besançon, France) performed initial sound-card records of the signal emitted by a vibrating tuning fork in order to identify some of the vibration modes. E. Rubiola and the members of the Time \& Frequency department of FEMTO-ST are acknowledged for motivating and fruitful discussions.

\section{REFERENCES}

${ }^{1}$ K. Kim, J. M. Lee, and Y. Hwang, Optics and Lasers in Engineering 46, 758 (2008).

${ }^{2}$ M. Helfrick, C. Niezrecki, P. Avitabile, and T. Schmidt, Mechanical Systems and Signal Processing 25, 917 (2011).

${ }^{3}$ W. Wang, J. Mottershead, T. Siebert, and A. Pipino, Mechanical Systems and Signal Processing 28, 333 (2012).

${ }^{4}$ D.-A. Wang, F.-W. Sheu, and Y.-S. Chiu, Optics and Lasers in Engineering 49, 954 (2011).

${ }^{5}$ D. Teyssieux, S. Euphrasie, and B. Cretin, Measurement 44, 2205 (2011).

${ }^{6}$ M. Ozbek, D. Rixen, O. Erne, and G. Sanow, Energy 35, 4802 (2010).

${ }^{7}$ P. Sandoz, R. Zeggari, L. Froehly, J. Pretet, and C. Mougin, J Microsc. 225, 293 (2007).

${ }^{8}$ R. Matsuzaki and A. Todoroki, Composites Science and Technology 66, 407 (2006).

${ }^{9}$ F. G. Márquez, A. Tobias, J. P. Pérez, and M. Papaelias, Renewable Energy 46, 169 (2012).

${ }^{10}$ Y. Zhang and J. Li, J. Comput. Civ. Eng. 20, 390 (2006).

${ }^{11}$ S. Kim, S. Pakzad, D. Culler, J. Demmel, G. Fenves, S. Glaser, and M. Turon, in 6th International Symposium on Information Processing in Sensor Networks(IPSN) (Cambridge, MA, 2007) pp. 254-263. 
${ }^{12}$ Y. Ni, B. Li, K. Lam, D. Zhu, Y. Wang, J. Lynch, and K. Law (2011) pp. 83-102.

${ }^{13}$ H. Hashemian, Annals of Nuclear Energy 38, 665 (2011).

${ }^{14}$ C. Gentile, Radar Technology, edited by G. Kouemou (Intech, 2009).

${ }^{15}$ J. Jeon, W. Hwang, H.-C. Park, and W.-S. Park, Composite Structures 63, 427 (2004).

${ }^{16}$ T. Thai, J.-M. Mehdi, F. Chebila, H. Aubert, P. Pons, G. DeJean, M. Tentzeris, and R. Plana, IEEE Sensor Journal 12, 2756 (2012).

${ }^{17}$ H. Aubert, F. Chebila, M. Jatlaoui, T. Thai, H. Hallil, A.Traille, S.Bouaziz, A. Rifaï, P. Pons, P. Menini, and M.Tentzeris, Annals of telecommunications 68, 425435 (2013).

${ }^{18}$ A. Pohl, R. Steindl, and L. Reindl, IEEE Transactions on Instrumentation and Measurement 48, 1041 (1999).

${ }^{19}$ D. Morgan, Surface acoustic wave filters. With applications to electronic communications and signal processing - 2nd Ed. (Academic Press, 2010).

${ }^{20}$ D. Mindell, Digital Apollo: Human and Machine in Spaceflight (The MIT Press, 2011).

${ }^{21}$ X. Bao, W. Burkhard, V. Varadan, and V. Varadan, Proc. of the IEEE Ultrasonic Symposium , 583 (1987).

${ }^{22}$ V. Plessky and L. Reindl, IEEE Trans Ultrason Ferroelectr Freq Control. 57, 654 (2010).

${ }^{23}$ A. Stelzer, M. Pichler, S. Scheiblhofer, and S. Schuster, IEEE Transactions on Ultrasonics, Ferroelectrics and Frequency Control 51, 1412 (2004).

${ }^{24}$ F. Schmidt, O. Sczesny, L. Reindl, and V. Mágori, Ultrasonics symposium , 589 (1994).

${ }^{25}$ H. Scherr, G. Scholl, F. Seifert, and R. Weigel, in IEEE Ultrason. Symp., Vol. 1 (1996) pp. $347-350$.

${ }^{26}$ R. Hütter and T. Ostertag, in Wireless Surface Acoustic Wave Sensor Symposium (Villach, Austria, 2012) pp. 1-27.

${ }^{27}$ M. Pieraccini, M. Fratini, F. Parrini, and C. Atzeni, IEEE Transactions on Geoscience and Remote Sensing 44, 3284 (2006).

${ }^{28}$ C. Gentile, NDT and E International 43, 231 (2010).

${ }^{29}$ J. Beckley, V. Kalinin, M. Lee, and K. Voliansky, in IEEE International Frequency Control Symposium and PDA Exhibition (2002) pp. 202-213.

${ }^{30}$ V. Kalinin, A. Leigh, A. Stopps, and E. Artigao, in Proc. IEEE IFCS (2013).

${ }^{31}$ R. Stoney, D. Geraghty, and G. E. O’Donnell, Int. J. of Automation Technology 7, 451.

${ }^{32}$ P. Sandoz, J.-M. Friedt, E. Carry, B.Trolard, and J. G. Reyes, American Journal of Physics 77, 20 (2009). 
${ }^{33}$ J. G. Zea, P. Sandoz, G. Laurent, L. L. Lemos, and C. Clevy, International Journal of Optomechatronics 7, 222 (2013).

${ }^{34}$ P. Sandoz, J.-M. Friedt, and E. Carry, Review of Scientific Instruments 78, 023706 (2007).

${ }^{35}$ J.-M. Friedt, C. Droit, S. Ballandras, S. Alzuaga, G. Martin, and P. Sandoz, Rev. Sci. Instrum. 83, 055001 (2012), available at http://jmfriedt.free.fr.

${ }^{36} \mathrm{~V}$. Kalinin, J. Beckley, and I. Makeev, in European Freq. \& Time Forum (2012) pp. 428-435.

${ }^{37}$ W.-E. Bulst, G. Fischerauer, and L. Reindl, IEEE Transactions on Industrial Electronics 48, 265 (2001).

${ }^{38}$ T. Rossing, D. Russel, and D. Brown, Am. J. Phys. 60, 620 (1992).

${ }^{39}$ Improved material constants for LiNbO3 and LiTaO3, Vol. 1 (Honolulu, HI, 1990). 


\section{LIST OF FIGURES}

1 (a) $800 \times 480$ subset of the $1024 \times 1024$ image of the periodic pattern used for tuning fork displacement measurement. During the calculation, a 1024-pixel long and 4 to 12-pixel wide horizontal swath of the image is selected with an

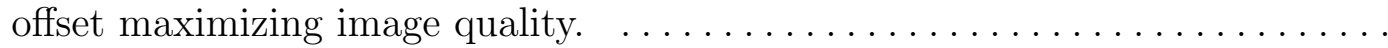

2 Phase, computed as $\arctan (Q / I)$, measured for various drive voltages of the voice coil. For the largest driving voltage, the phase rotation of $2 \pi$ is visible (blue curve). Tuning the phase offset $\varphi_{0}$ maximizes the measurement range by keeping the phase rotation within the $[-\pi / 2, \pi / 2]$ range. . . . . . . . . .

3 Impulse reponse of the tuning fork as observed using image processing: a frame rate of 22779 images/s is achieved by restricting the region of interest with to a 1024-long, 4-pixel wide swath. Two modes around $440 \mathrm{~Hz}$ and $10 \mathrm{kHz}$ are visible. Insets: time domain-measurement of the exponentially decaying vibration amplitude of the impulse response (top), and (bottom) zoom on the initial impulse response in which two-mode contribution is visible. 21 Comparison of the displacement measurement using the optical in-plane detection method (bottom curves), and the acoustic resonator acting as a strain gauge measurement (top curves) at a $7 \mathrm{kS} / \mathrm{s}$ sampling rate. Left: driving voltage of the voice coil is $2 \mathrm{~V}_{p p}$. Right: driving voltage of the voice coil is $10 \mathrm{~V}_{p p}$. $S_{11}$ frequency (top) and time (bottom) domain responses of the acoustic delay

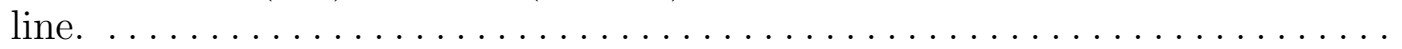

6 Schematic of the experimental setup for probing an acoustic delay line response at 250 ksamples/s. . . . . . . . . . . . . . . . . . . . .

(a) Time-domain characterization of the impulse response of the acoustic delay line. The arrow indicates the echo analyzed for measuring the strain as the delay line was glued on a tuning fork. Time ranges from 0 to $4.096 \mu \mathrm{s}$. (b) Time-domain evolution of the I and Q components of the echo under investigation. Samples are separated by $4.096 \mu$ s so the total duration of this 2048-sample record is $8.39 \mathrm{~ms}$. The tuning fork is excited at $443 \mathrm{~Hz}$, or a 2.25 ms period: 3.7 periods are expected. . . . . . . . . . . . . .

8 Fourier transform of the impulse response of the tuning fork when hit to generate a broadband response: all peaks $(443 \mathrm{~Hz}, 5465 \mathrm{~Hz}, 10143 \mathrm{~Hz}$ and $40714 \mathrm{~Hz}$ ) are attributed to tuning fork modes since the background spectrum has been measured as a constant noise below a background level of 280 arbitrary units, or 4-times lower than the smallest visible peak in the Fourier transform of the impulse response record. Red is the Fourier transform of the $\mathrm{Q}$ component, blue is the Fourier transform of the I component. Inset: time-domain record exhibiting the exponentially decaying oscillation of the tuning fork (top), and zoom on the first periods in which the combination of

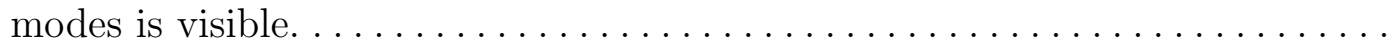

Evolution of the phase standard deviation, first driven by the local oscillator phase noise and then by the low noise amplifier on the receiver stage once the returned power becomes too low for the phase noise of the amplifier to become dominant. Left axis: phase standard deviation. Right axis: the phase standard deviation is converted to a strain standard deviation assuming a $\mathrm{LiNbO}_{3} \mathrm{YXl} / 128^{\circ}$ substrate and an at echo $1 \mu \mathrm{s} . \ldots \ldots \ldots \ldots \ldots \ldots$ 


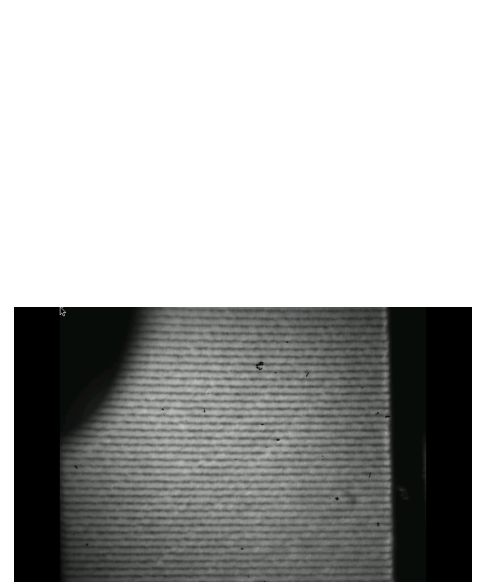

(a)
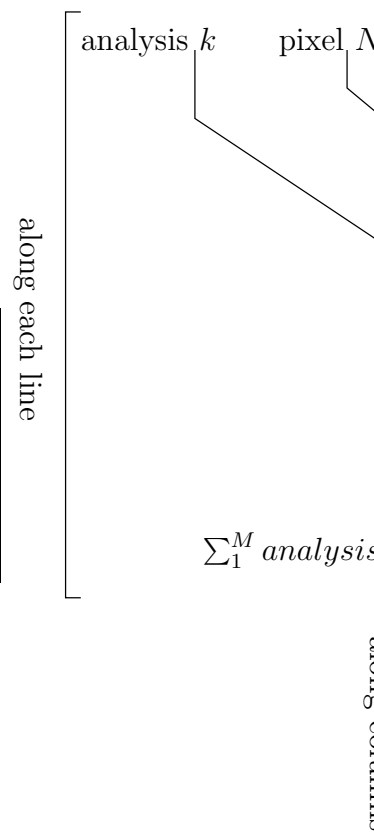

(b)

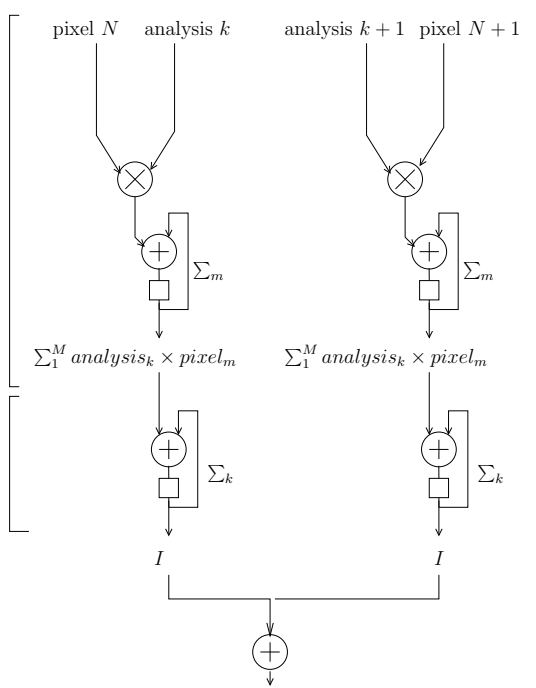

(c)

FIG. 1. (a) $800 \times 480$ subset of the $1024 \times 1024$ image of the periodic pattern used for tuning fork displacement measurement. During the calculation, a 1024-pixel long and 4 to 12-pixel wide horizontal swath of the image is selected with an offset maximizing image quality. 


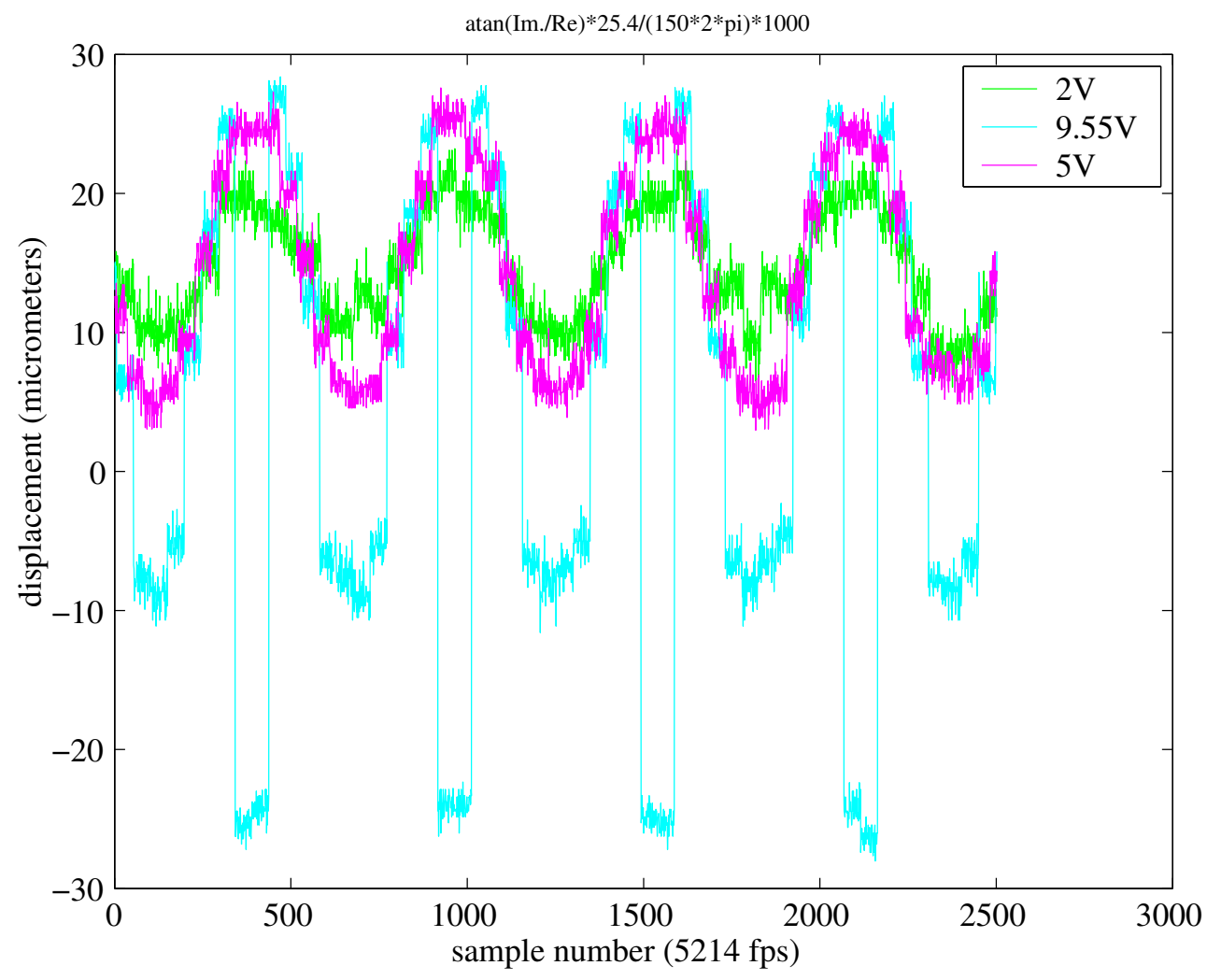

FIG. 2. Phase, computed as $\arctan (Q / I)$, measured for various drive voltages of the voice coil. For the largest driving voltage, the phase rotation of $2 \pi$ is visible (blue curve). Tuning the phase offset $\varphi_{0}$ maximizes the measurement range by keeping the phase rotation within the $[-\pi / 2, \pi / 2]$ range. 


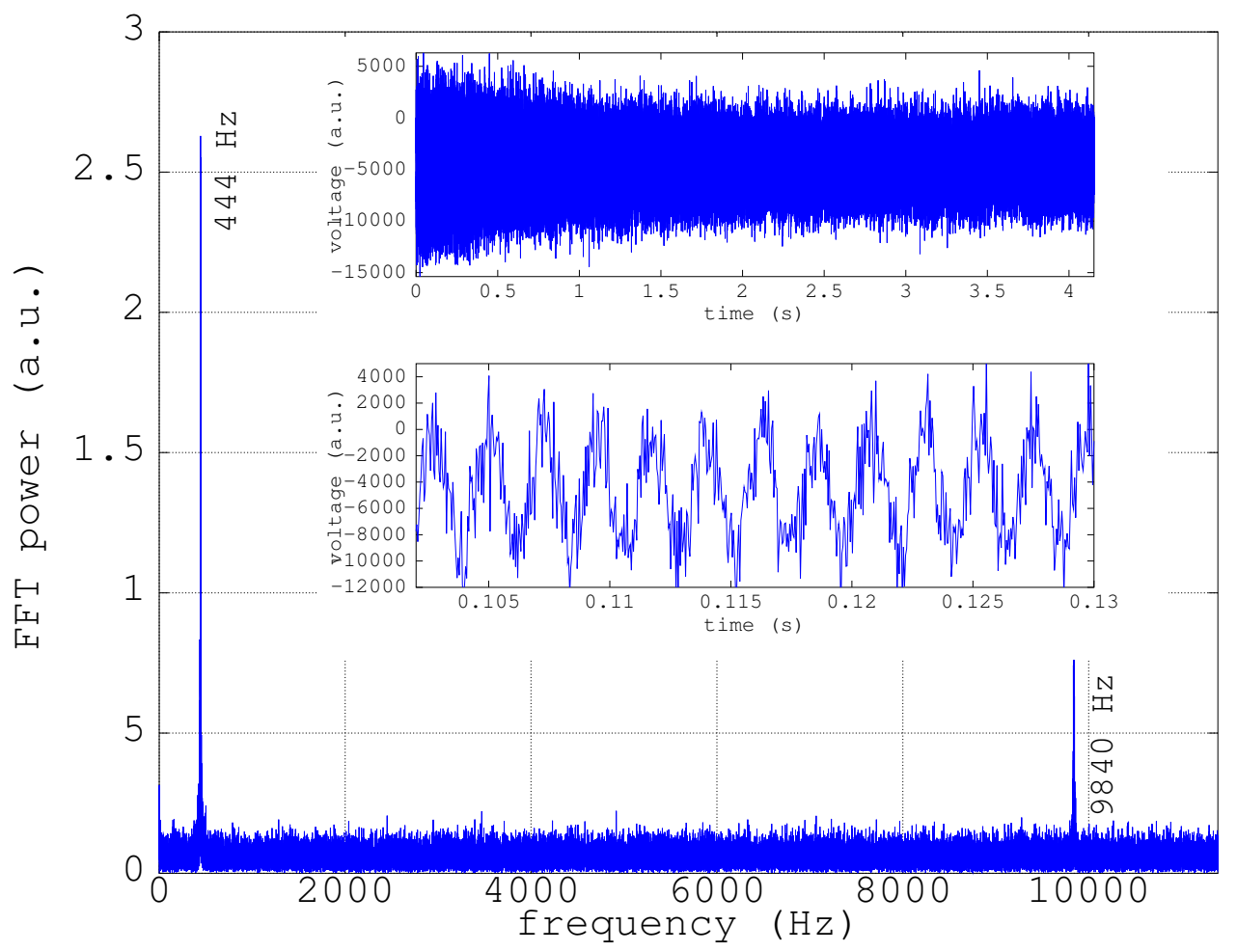

FIG. 3. Impulse reponse of the tuning fork as observed using image processing: a frame rate of 22779 images/s is achieved by restricting the region of interest with to a 1024-long, 4-pixel wide swath. Two modes around $440 \mathrm{~Hz}$ and $10 \mathrm{kHz}$ are visible. Insets: time domain-measurement of the exponentially decaying vibration amplitude of the impulse response (top), and (bottom) zoom on the initial impulse response in which two-mode contribution is visible. 

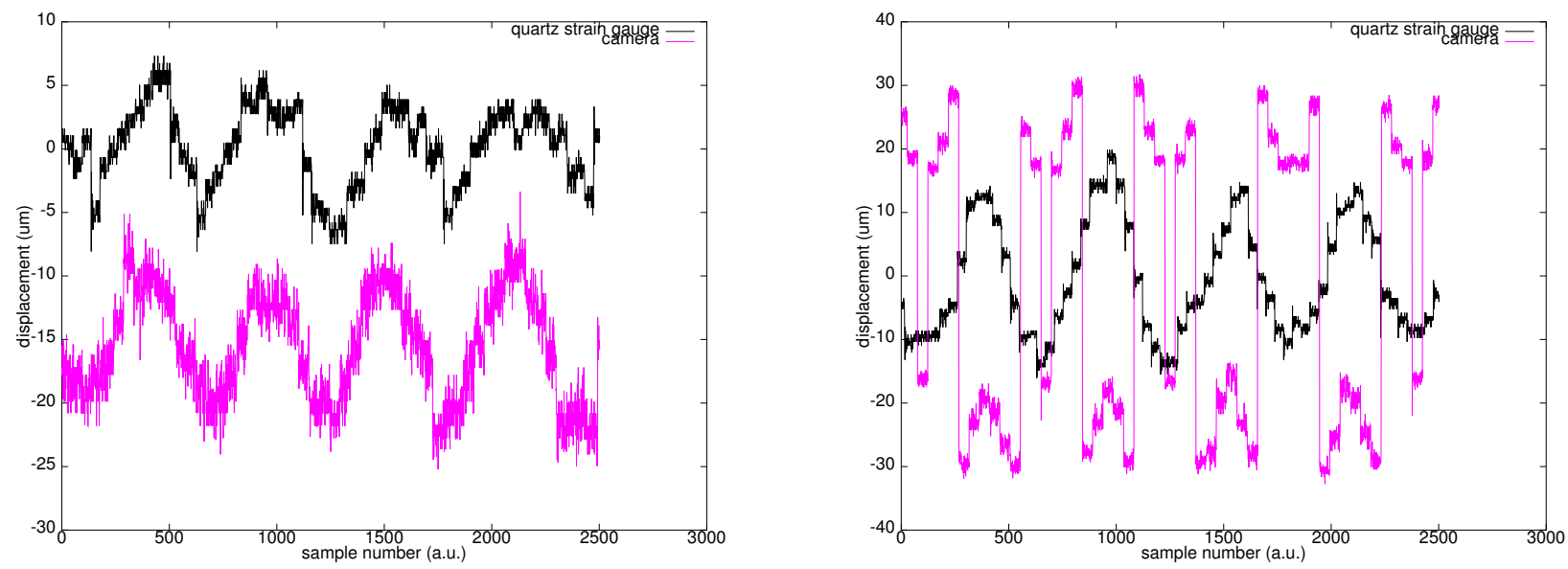

FIG. 4. Comparison of the displacement measurement using the optical in-plane detection method (bottom curves), and the acoustic resonator acting as a strain gauge measurement (top curves) at a $7 \mathrm{kS} / \mathrm{s}$ sampling rate. Left: driving voltage of the voice coil is $2 \mathrm{~V}_{p p}$. Right: driving voltage of the voice coil is $10 \mathrm{~V}_{p p}$. 

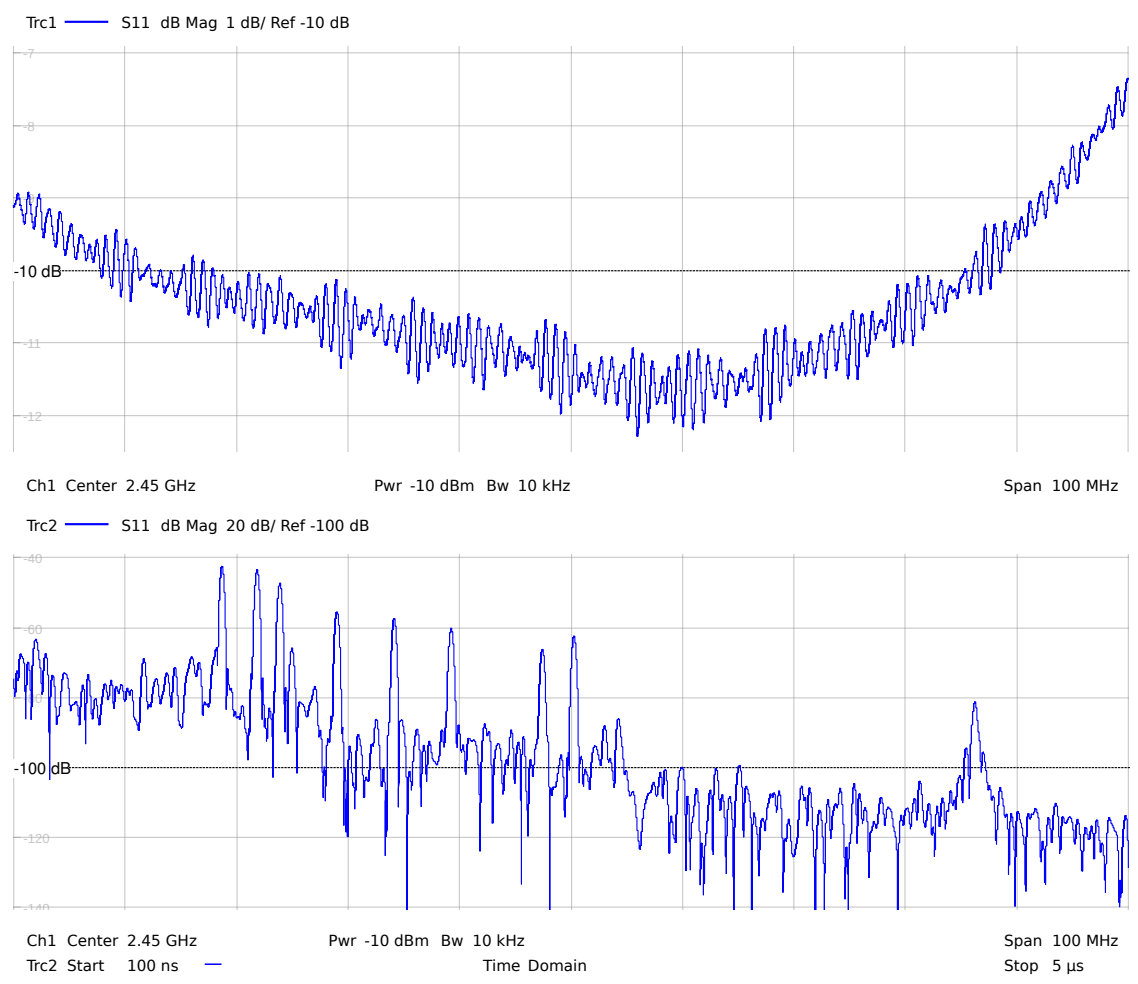

FIG. 5. $S_{11}$ frequency (top) and time (bottom) domain responses of the acoustic delay line. 


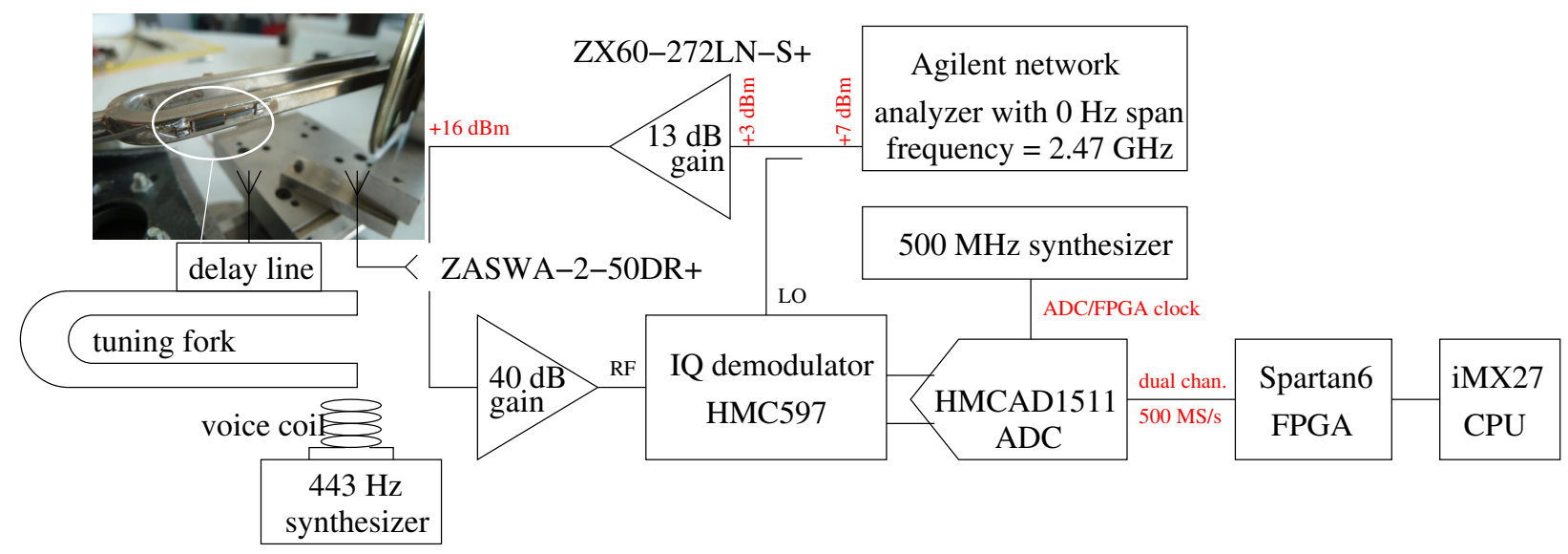

FIG. 6. Schematic of the experimental setup for probing an acoustic delay line response at 250 ksamples/s. 


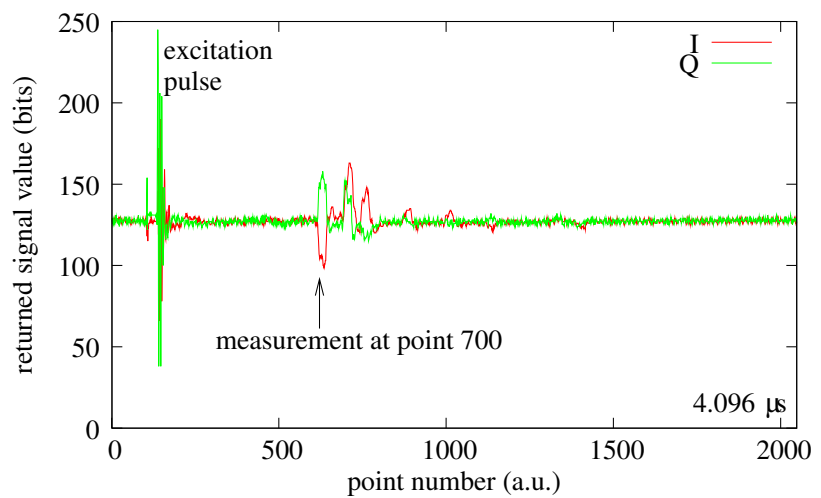

(a)

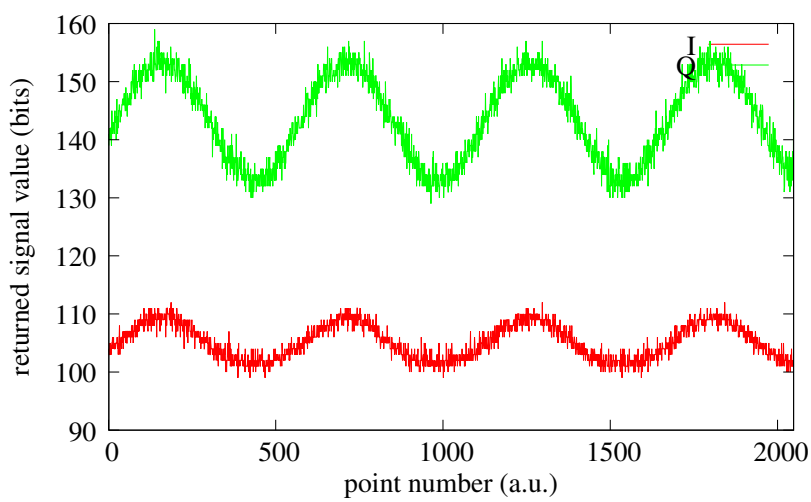

(b)

FIG. 7. (a) Time-domain characterization of the impulse response of the acoustic delay line. The arrow indicates the echo analyzed for measuring the strain as the delay line was glued on a tuning fork. Time ranges from 0 to $4.096 \mu \mathrm{s}$. (b) Time-domain evolution of the I and Q components of the echo under investigation. Samples are separated by $4.096 \mu$ s so the total duration of this 2048sample record is $8.39 \mathrm{~ms}$. The tuning fork is excited at $443 \mathrm{~Hz}$, or a $2.25 \mathrm{~ms}$ period: 3.7 periods are expected. 


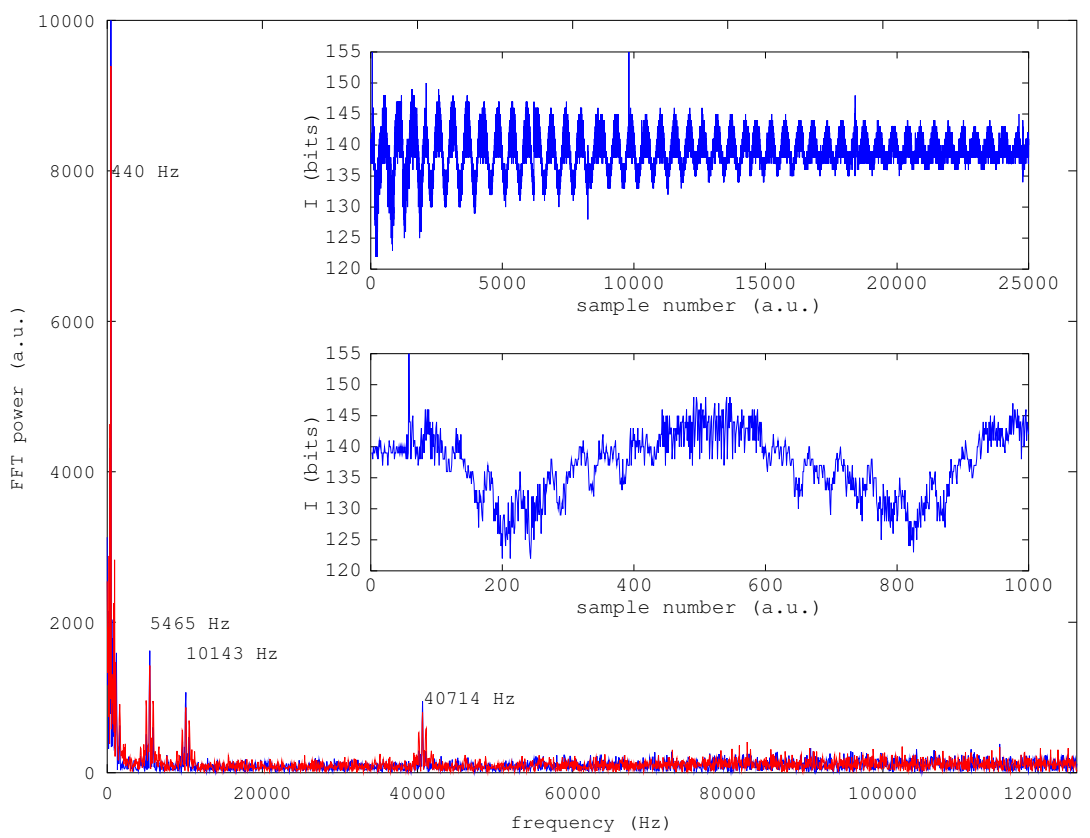

FIG. 8. Fourier transform of the impulse response of the tuning fork when hit to generate a broadband response: all peaks $(443 \mathrm{~Hz}, 5465 \mathrm{~Hz}, 10143 \mathrm{~Hz}$ and $40714 \mathrm{~Hz}$ ) are attributed to tuning fork modes since the background spectrum has been measured as a constant noise below a background level of 280 arbitrary units, or 4-times lower than the smallest visible peak in the Fourier transform of the impulse response record. Red is the Fourier transform of the Q component, blue is the Fourier transform of the I component. Inset: time-domain record exhibiting the exponentially decaying oscillation of the tuning fork (top), and zoom on the first periods in which the combination of modes is visible. 


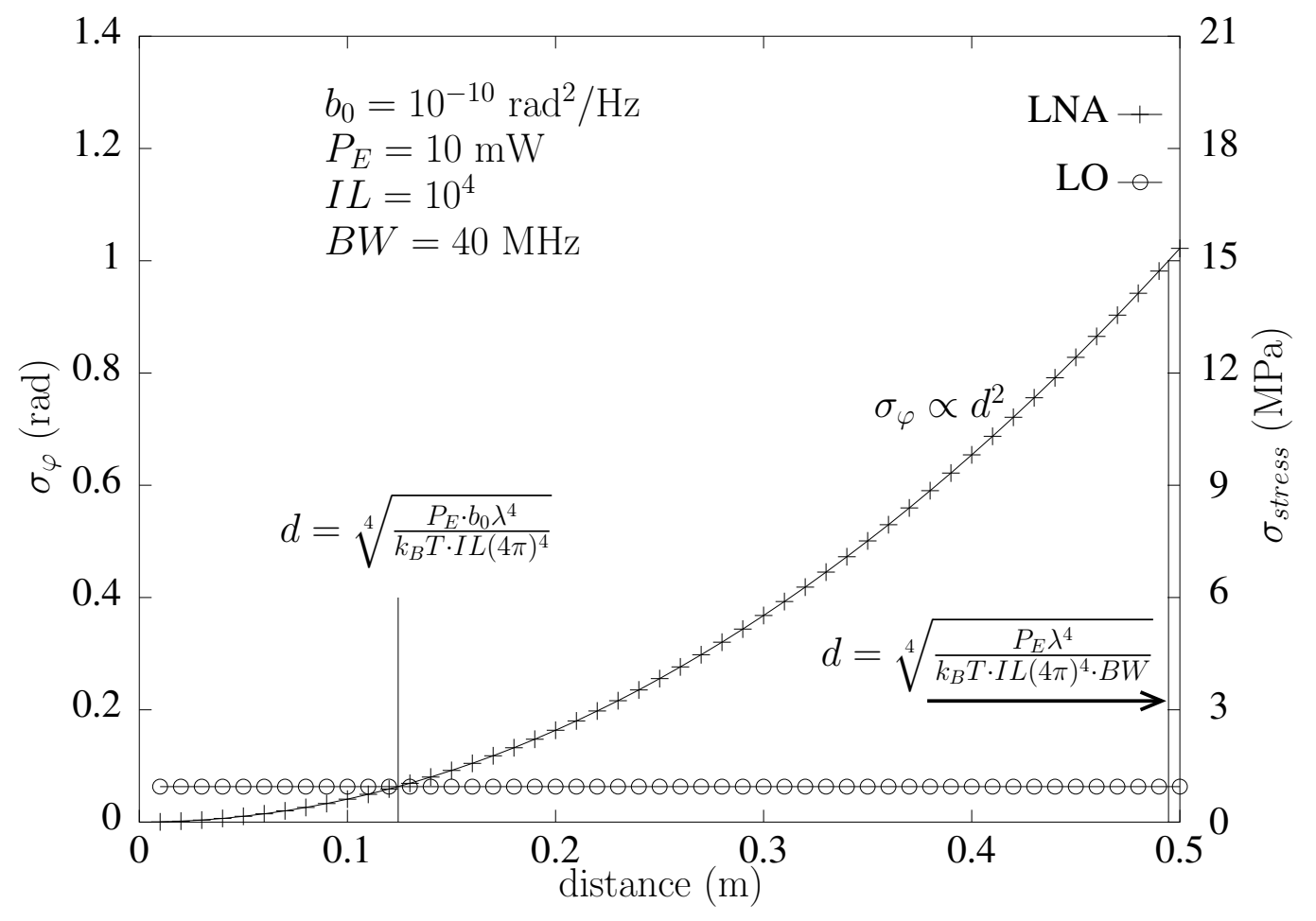

FIG. 9. Evolution of the phase standard deviation, first driven by the local oscillator phase noise and then by the low noise amplifier on the receiver stage once the returned power becomes too low for the phase noise of the amplifier to become dominant. Left axis: phase standard deviation. Right axis: the phase standard deviation is converted to a strain standard deviation assuming a $\mathrm{LiNbO}_{3} \mathrm{YXl} / 128^{\circ}$ substrate and an at echo $1 \mu \mathrm{s}$. 